\title{
Mobile Interface Design to suit the Algerian Culture: First initial design
}

\author{
Brahim Abdesselam, Madihah Sheikh Abdul Aziz \\ Department of Information Systems, Kulliyyah of Information and Communication Technology, \\ International Islamic University Malaysia, Gombak, Selangor, Malaysia. \\ madihahs@iium.edu.my
}

\begin{abstract}
The growing number on the use of mobile apps has extraordinary potential for the public transport industry. With mobile apps, may provide fast, effective, and safe services to passengers commuting to different destinations. However, the design of mobile applications needs particular attention concerning the usability aspects, such as culture. In Algeria, Taxi-Inter-Wilaya is a shared-taxi service between cities. This taxi service is estimated as one of the most beneficial ways for the Algerian to travel. However, the booking for passengers travelling to long distances of $600 \mathrm{KM}$ and more, can only be made via phone calls. Consequently, passengers face various challenges in the booking process. Drivers receive calls for bookings while driving, which puts them in a dangerous situation. To this aim, the researcher uses the design thinking approach to design a transport mobile application that can support both taxi drivers and passengers to travel between cities suitable to the Algerian culture. The data collection method comprises a series of interviews with both users (passengers and taxi drivers) as well as designing several iterations of prototypes of the mobile application, named DJAMAAI. The prototypes were then evaluated via a series of usability testing for both groups of users. Results show that users favour visual representations that are associated to their culture more than textual; they prefer, buttons such as icons and symbols, as well as more straightforward and minimalist user interface design. Also, symbols and hints help learnability of using the application.
\end{abstract}

Keywords— Usability Testing, Design Thinking, Mobile Interface Design, Culture

\section{INTRODUCTION}

Algeria is the tenth-largest country in the world after Kazakhstan [1]. Also, it is the largest in Africa and the Mediterranean region [2]. It has a diverse transportation system which includes many transportation infrastructures. In addition to that, there are multiple ways to travel between cities; the passenger can use the airlines, shuttle buses, minibuses, or taxies between cities that are also known as "Taxi-Inter-Wilaya" [2].

Transport overland is the cheapest. Trans-Sahara Highway is the nearest and the best way to travel from the north to the south of Algeria. It passes through Ghardaia city which is the main road to enter the Algerian Sahara. Ghardaia is situated at the centre of the country and constitutes as a hub to travel to the four edges of the country [3].

Taxi-Inter-Wilaya is a shared taxi service. It is a useful way to travel for long distances, and it is a commonly used transport to travel amongst cities in Algeria [4]. This taxi service fetches customers at the taxi-rank. A taxi-rank or taxi-stand is the place where taxis queue for customers or vice-versa. These taxi-ranks are usually located outside of essential transport hubs, e.g. at the airports, or railway stations. They are organised based on first-in-first-out, however, there is also, a tendency for passengers to choose the first taxi in the rank [5].

Shared taxi service is a form of public transport between regular taxi and bus services. Taxi sharing is common in many developing countries, also in some developed countries as well [5]. It can be categorised as a separate segment or as part of regular public transport. This sharedtaxi service operates along a pre-defined line like unscheduled bus service [5]. Due to its predefined city of departure and city of arrival, the price of the journey is usually fixed in advanced [5]. These taxis which travel to distant cities on a trip of eight hours and more, are not able to go to the taxi-rank, but passengers may book their seats via phone calls.

\section{A. Mobile Application for Public Transport in Algeria}

Algeria seeks to benefit more from technology. The country acknowledges the need for technology and supports any development that contributes to the country. Since 2017, many applications have emerged in the taxi services industry. The applications are now available in the Algerian market, such as Coursa, Yassir, TemTem [6-7], Wesselni, Careem, Rekba, and Atlaa [7].

Overall, these applications work in a similar geographic region, which is in the urban zone [8], and they focus only on hailing taxi service. Besides, they use the dynamic pricing 
process to evaluate the trip cost which leads to expensive long trips. For example, the ride from Ghardaia to Algiers (two different cities) in shared-taxi service costs 1700.00DZD, but the same trip was estimated by Yassir (one of the applications available) as 18010.00DZD. This is approximately ten times the cost in Taxi-Inter-Wilaya [9]. Moreover, the price of a planned trip is high compared to the un-planned trip. These factors deny Taxi-Inter-Wilaya to benefits from the existing Taxi mobile apps.

The booking process in the existing Taxi-Inter-Wilaya services are lengthy and complex [10], whereby a passenger needs to call the driver via phones to ask for information about the schedule of the trip, the available seats, to make a new booking, and to confirm or to cancel the old booking. It is also almost impossible for the passenger to know a driver's service performance and behaviour due to the unavailability of such information. In addition to that, a driver may receive phone calls at any time, and sometimes they must accept calls and taking new bookings while driving other passengers. Consequently, drivers may mishandle the situation such as forgetting the booking or cancellation as well as forgetting the details of a passenger, as there is no effective method to record the details. More importantly, answering phone calls while driving is illegal, unsafe, and susceptible to road accidents. To our knowledge, it was hard to find a robust system or application to administer these Taxi-Inter-Wilaya services for the benefits of both drivers and passengers in Algeria.

\section{B. Cultural-related Mobile Interface Design}

One aspect of design is culture. Algeria is an Arab country, and Arabic is the first language of the Algerian people. This gives more opportunity for mobile applications with an Arabic version as it is understandable and popular in the country. However, the non-Arabic version of apps mostly were unsuitable for the Arabs because of the orientation of the user interface (left-to-right) which makes it complicated and unfamiliar to the users [11]. The existing taxi service mobile applications failed to provide language options for example Arabic language because Algerian people speak both Arabic and French languages [12]. In addition, no information on gender was provided in the booking process. With a long-distance journey, passengers often choose their preferred seat in a car, and refuses to change them if requested by others, for example a female passenger that usually prefers to be seated with the same gender. Moreover, the existing applications are designed in black or red colours. In Algeria, Black is regarded as a symbol of evil and death, while Red is the symbol of danger and blood [13].

Most of the existing applications originated from the west. Therefore, the design was based on, for example the European culture, whereby lack of the products' analysis is directed to a targeted culture [14]. This western designed interface provokes difficulty to the users in another context of use. This can be supported with an earlier research by Nisbett [15] who suggested that geographical differences cause Westerners and Easterners to think and perceive information differently. As an example, while, the European prefers textual information, the eastern prefers graphics [16-17]. A research by Shen et al. [18] share similar findings where the westerners favour left to right, top to bottom layout, as compared to traditional eastern culture, they are familiar with right to left writing which determines their design preferences. The overall finding demonstrates that distinct cultural background leads to different perspectives, values and norms which may affect the user interface design [14].

The focus of this research is to address the challenge associated with Taxi-Inter-Wilaya services and passengers in Algeria and to design a mobile application that can assist and support both drivers and passengers to efficiently and effectively commute between cities, cost effective and ultimately, to have a pleasant experience suitable to Algerian culture.

\section{METHODOLOGY}

This study proposed the use of the Design Thinking Method, to design the mobile application to suit the Algerian culture. This approach gives a solution-based strategy to solve the problem at hand. It is beneficial in treating complicated problems that are ill-defined or unexplored, by identifying the human needs included, by reframing the difficulty in human-centric processes, by building multiple ideas in brainstorming sessions, and by using a hands-on way in prototyping and testing [19].

There are five necessary activities in design thinking. The process includes 1) empathise: to understand user's needs, 2) define: to define, explore and establish problems, 3) ideate: to propose new possible ideas, 4) Prototyping; to put the collected solution in a prototype, and finally 5) Testing; to get feedback from the actual users. Design thinking is a human-centred approach that comprehensively and iteratively evaluating what is being built, with the involvement of users, throughout the process from the beginning through to the end, with the ultimate target, providing better user experience [19].

\section{A. Participants and Procedure}

Following the first phase, emphasise: the study started by conducting a series of informal interview with a group of twenty passengers and twenty taxi drivers [20]. They were interviewed in individual sessions. The recruited participants were between the ages of 18 to 60 and are smartphone users. The passengers were frequent commuters from Ghardaia to Algiers, Oran, or Constantine, among the cities in Algeria. The taxi drivers were permanently commuting between Ghardaia and to one of 
the three Algerian cities. The interviews took place in quiet places where the interviewer felt comfortable.

Upon completion, the study then proceeds to the Define stage, where problems have been identified and analysed through thematic analysis.

Based on the problems identified, ideas were generated in the third stage, as a proposed solution by creating several possible sketches before proceeding to the prototype development stage. The ideas were then communicated to the users through informal interviews, to further explore their opinions.

Next, the study then continues with the development of the prototypes (stage 4 of the Design Thinking approach) after receiving feedback from the users, in the Ideate stage.

The prototypes were first design on a paper-and-pencil sketches, to gain as much feedback as possible from the users. Paper-and-pencil sketches (low-fidelity prototypes) are used widely during the design process due to their low cost and efficiency in getting feedback from the actual users. The sketches can be used to develop, explore, communicate, and evaluate the designer's ideas [21-22]. Paper prototypes help in getting substantive user feedback, promote rapid iterative development, and allow for easy and inexpensive modifications.

The Design thinking approach promotes the idea of getting users' feedback in each stage, to ensure that all angles of design are suitable, and users can understand them well. Upon completion of the prototyping stage, the Usability Testing was carried out in two iterations to gain further feedback from both groups of users. This paper focuses on the Usability Testing of the two prototypes that have been evaluated. The next section discussed the analysis of the testing.

Upon arriving at the chosen area, the researcher welcomed and briefed participants on the purpose of the evaluation, emphasising that a Usability Test aims to assess the usability of the DJAMAAI App - not to evaluate the participants -. Next, they read the relevant cover story and then they attempted the tasks, one at a time, at their own pace. The participants were asked to verbalise their thoughts as they went; the researcher took notes throughout. Upon completion of all the tasks, the participants answered three questions verbally and responded to the SUS questionnaire. Finally, they were thanked and excused.

To evaluate the proposed prototype, each participant was given a set of scenarios to evaluate the proposed prototype. The scripts were written clearly for each group of the passenger or the taxi driver.

To determine the success of the DJAMAAI App, usability goals were set in terms of the number of questions, hints and errors allowed according to tasks during the usability tests. The numbers in the goals were based on the author's best guess of the degree of difficulty associated with each task.

For a hint to be given, the participants were allowed 3-5 minutes to perform a task, depending on the task in question. If a participant would get stuck and did not ask any question, the researcher gave one hint. For example, of passengers, after choosing a city of destination in a new booking, some participants needed to be reminded: "to each trip destination city and departure city".

\section{DATA ANALYSIS}

Problems identified during the interview in the earlier stage of the data collection, were further analysed and defined. This study proposed a mobile application called DJAMMAI, that contains two modules, to cater for both passengers and taxi drivers. Two initial prototypes for both drivers and passengers were created and tested through a formal method of Usability Testing. The testing is conducted to identify and eliminate any usability flaws from the earliest stage of prototype design.

After running a pilot test to ensure a smooth running of the test with five users, the actual test was then started. Twenty participants (10 Passengers and 10 Taxi drivers) evaluated the low-fidelity prototypes in two iterations, LowFidelity1 and Low-Fidelity2 [23].

During the tests, participants were given scenarios and tasks. While the participants were performing the tasks, their performances were observed, such as time spent, steps taken, questions asked, hints given were all noted for further analysis [24]. Upon completing the tasks, they were asked to express their opinion regarding the prototype and to respond to the usability questionnaires using SUS System Usability Scale [25] The survey responses will be used to determine the score of Usability aspect of the application.

For Low-fidelity 1, a total of five usability issues and four suggestions were discovered from the passengers' group. Meanwhile, a total of twelve usability issues were discovered from the drivers' group.

Of these, seven were incomprehensible symbols, six on poor user interface design, and two lack of necessary information pointed out by the participants. On the other hand, the study able to capture users' attention and provides the researcher with essential observations to improve the prototypes. Some of the suggestions were also taken into consideration in the next refinement of the prototypes. The next section discussed the results.

As for the Low-Fidelity 2, at least three usability issues and three suggestions were identified in the passengers' group. A decrease in the number of usability problems found in the second prototypes suggest a positive improvement of the prototypes. Similarly, for the taxi drivers' group, only two usability issues and three suggestions found. 


\section{DISCUSSION OF RESULTS}

\section{B. Low-fidelity 1}

For the passengers, the participants asked two questions, mainly about the "Departure-cities UI", the way of choosing a trip offer, the method of getting to the taxi driver details, and the way of getting to trip's properties. A total of two hints were given. One concerned the New-booking task, and one in the Rating-driver. Two errors occurred in the Tripdetails, including participants mistakenly selecting a trip of two ways instead of one way, and filling the wrong field of date because they look similar.

For the taxi drivers, the participants asked two questions, mainly about the meaning and location of the new booking's icon, the payment's icon, and the booking management's icons. A total of two hints were given; they were associated with the Manual-new-bookings task. Two errors occurred in Manual-new-bookings task, so they failed to complete the operations (new booking, and payment) because they did not recognise the symbols used or they did not find them. The observation here can be related to the importance on the use of accurate and clear symbols or icons in the design of user interface, to ease navigation processes across applications. The design of symbols and icons need to be suitable with the culture of the Algerian people, to give them a sense of familiarity.

\section{Participants' Opinion}

For the passengers, in response to Question 1, concerning what the participants think about these prototypes, three participants expressed that the tool can be interesting, and might be able to improve the booking of seats for a long journey. Two participants were concerned about the taxi driver behaviour, and they said: "Such a tool will make taxi drivers think deep (being more careful) in their behaviour towards passengers".

In response to Question 2, concerns harder parts in the prototypes, three participants mentioned the complexity in a new booking seat. Suggesting further refinement needed on this part.

In response to Question 3, concerning what they liked the most; all participants basically like all area and did not share any specific part. However, they insisted on making it more manageable and usable, as they felt that the user-interface design was complex.

The SUS average score was 68.5 indicates that participants are dissatisfied with the proposed prototype. This can be explained with several errors made and questions asked by the participants in the first session of the formative usability testing. Some suggestions include, 1) Reorganising of information on $\mathrm{UI}$ in a more logical manner based on the users' input, 2) Addition of hints/information on the UI. Further refinement on the interface have been done, and tested in the Low-fidelity 2 prototypes.

For the taxi drivers, in response to Question 1, concerning what the participants think about these prototypes, three participants felt that that the tool can be useful and suggested to improve the management of seat's bookings. Two participants added that this tool will make the taxi drivers' job better with less disruption, particularly during driving.

In response to Question 2, concerning the difficult part, four participants highlighted the booking component that needs to be refined further. All drivers also shared similar opinion with regard to Question 3 with the passengers' group, whereby they thought that the UI of the prototypes is complex, and suggest some ways to improve and make it easier to use, from their perspectives. All relevant ideas, suggestions, usability issues were recorded, and referred to when refining the prototypes.

The SUS average score was 78.5, suggest that the initial design prototypes for the group of taxi drivers is accepted.

The improvements and amendments that were made to the prototype as suggested by the participants were "trip flexible research" which provides more offers during weekdays in the passenger's module. Another example is to add titles and graphic symbols to the user-interface. As highlighted in the literature, choosing symbols or graphical representations that are clearer and relevant to the context of use is vital, or they might lead to a wrong understanding and interpretation [26-27].

\section{Low-fidelity 2}

After refining the DJAMAAI App's prototype based on the users' input, following are some examples of changes made from Low-fidelity 1 to Low-fidelity 2. 


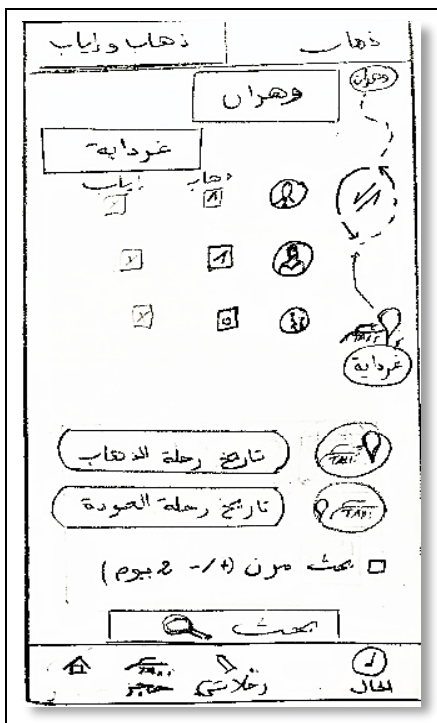

Low-fidelity 1

Three participants forgot to fill in the field of departure city in Research-trip-details. The participants went directly to fill in the number of passengers.

\section{Suggestion}

Two participants suggested to add titles at the top of each screen.

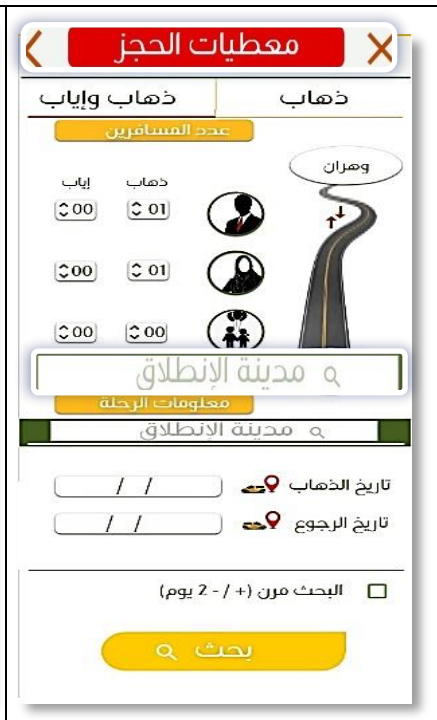

\section{Low-fidelity 2}

The information on the Researchtrip-details UI were reorganised.

Titles were added at the top of each screen.

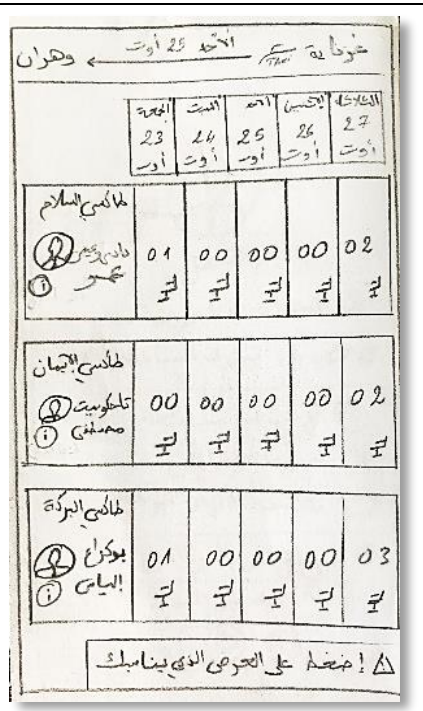

Low-fidelity 1

1 Usability issue

Three participants picked a

wrong choice of offer from the Trip-offers interface.

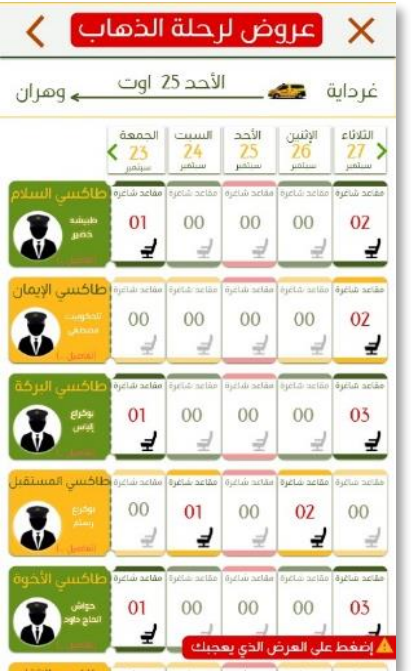

Low-fidelity 2

Colours were used to give better idea between an offer and the trip's date

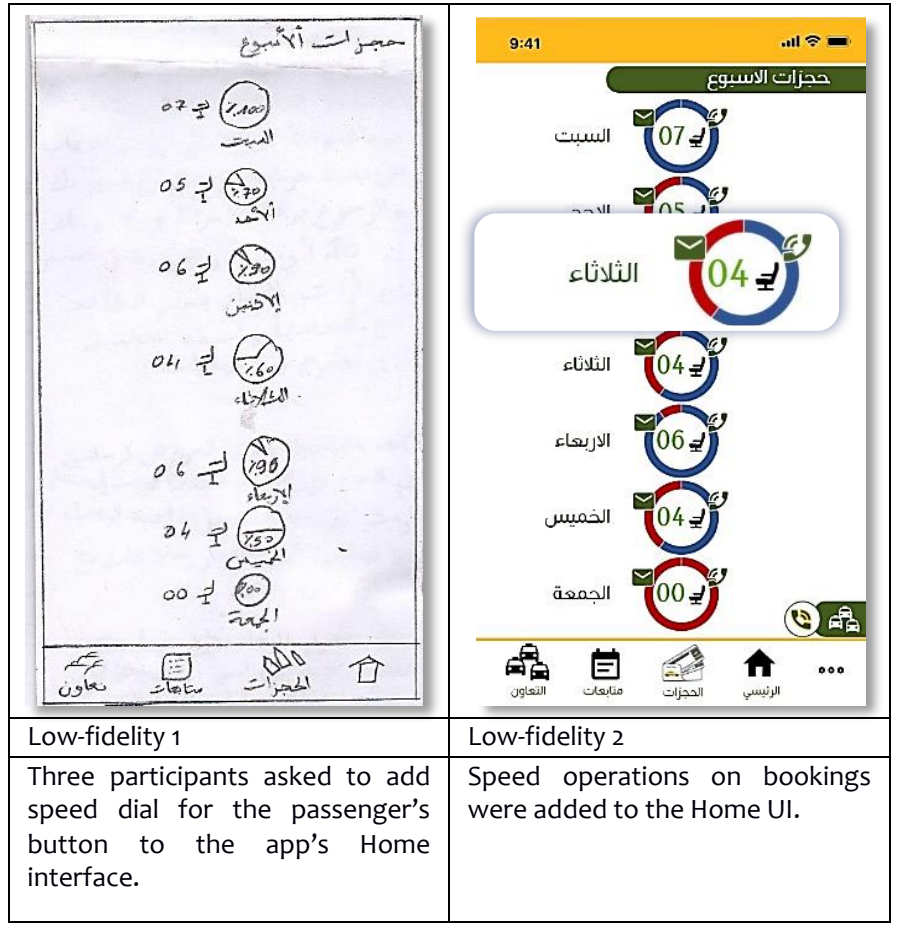

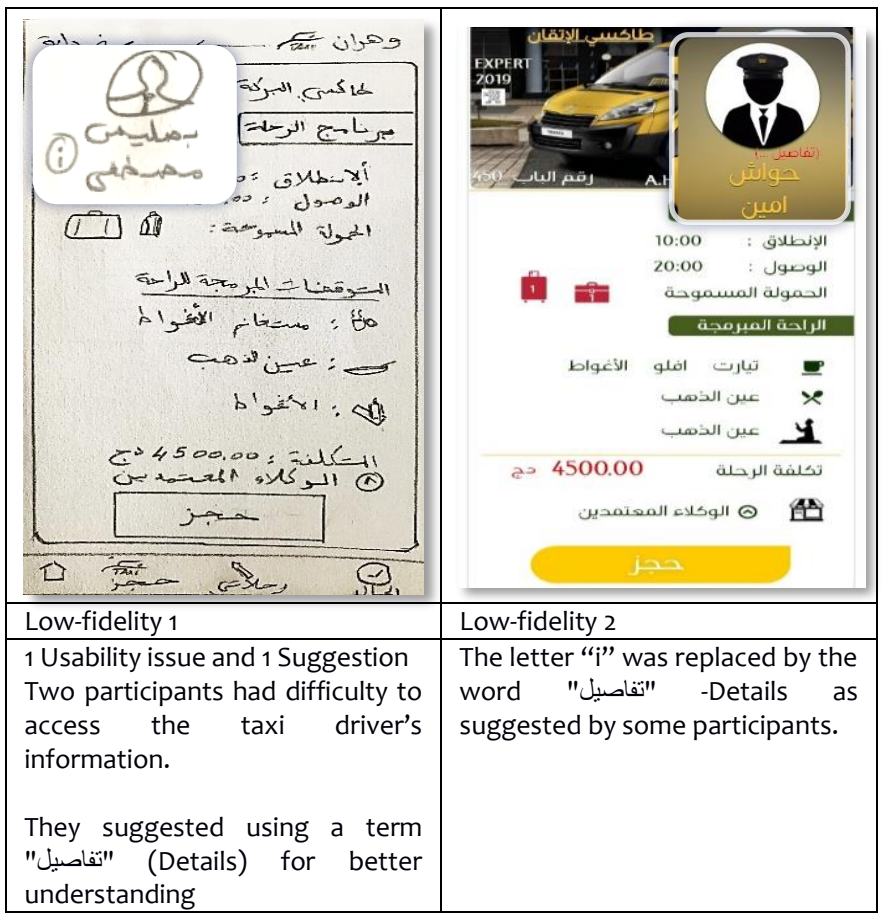


The results of the second iteration of the low-fidelity prototypes improves significantly, with Average score of SUS to 80 for passengers, and 83.5 for the drivers. Both groups of users like the refined look, with new additions that were added based on the first test.

In the low-fidelity 2 test, when asked which part/area of the app that was the hardest, all participants referred to the Bookings component, and two pointed to the Collaboration component.

When asked the part that they liked the most, all passengers referred to the management of booking and details of a passenger. Three participants also like the Collaboration part, that they felt was impressive, allowing the taxi drivers to collaborate with other drivers to improve the service. However, this part needs further refinement, as commented earlier, that it was difficult to navigate through.

Another observation, it was noticeable throughout the tests, that when participants search for information while performing the tasks, they always start from the right side to left of the interface and from the top to bottom, suggesting the different style of reading as opposed to the western culture. The design of this prototype follows the Middle East Culture, reading from right to left. Additionally, when they wanted to understand the meaning of symbols or icons used in the prototypes, they looked for any hint that could indicate and facilitate the understanding of the ambiguities. Likewise, as for taxi drivers, when they wanted to confirm a passenger's seat; they explored the day trip that is located under the heading "Reservations of the week", then finding the name of the passenger. It was observed that they tried to identify the symbol that indicates the meaning of "seat confirmation". This observation suggests the usefulness of symbols used in the design of this prototypes, according to their culture.

All observations that include, hints given, questions asked, errors made, and suggestions given by the participants in the Low-fidelity Test 2 were then recorded and used in the next refinement of high-fidelity prototypes. The design and evaluation of the prototypes will be presented and discussed further in the next publication.

\section{CONCLUSION}

Throughout these two iterations of the usability study, it appeared that the symbols used in the proposed prototypes have a significant role in simplifying the understanding of the user interface, thus provide a higher degree of learnability and usability. Also, all information necessitated by the users make the app more valuable to them, suits their needs and ultimately their culture. As for the conclusion, the low-fidelity prototypes ( 1 and 2 ) proposed in this initial stage of the study shows high acceptance level among users because they see that this proposed app can solve some of the difficulties they face when using the Taxi-Inter-Wilaya. The tool may help them to travel between cities efficiently and effectively.

The future work is to refine the prototypes based on the feedback gathered in this first formative testing and to be evaluated through another series of usability testing. The final findings of the study will be published in the next publication.

\section{ACKNOWLEDGEMENTS}

We would like to thank the passengers and the taxi drivers from Ghardaia MZAB who have volunteered to participate in this research.

\section{REFERENCES}

[1] M. Mattyasovszky, The Largest Countries in the World WorldAtlas.com. Retrieved April 12, 2019, from 16/12/2019 website: https://www.worldatlas.com/articles/the-largest-countries-in-theworld-the-biggest-nations-as-determined-by-total-land-area.html, 2019.

[2] Encyclopædia Britannica, I, Algeria. Retrieved from Encyclopædia Britannica, inc. website: https://www.britannica.com/place/Algeria, 2019.

[3] Expertalgeria, Spotlight on the Sahara | Algeria Tourist Attractions | Expert Algeria. Retrieved May 4, 2019, from https://www.expertalgeria.com/spotlight-sahara.html, 2019.

[4] A. Routard, Algérie | Transports et déplacements | Routard.com. Retrieved March 4, 2019, from

https://www.routard.com/guide/algerie/2121/transports.htm, 2019

[5] J. Aarhaug, Taxis as a Part of Public Transport. Deustsche Gessellschaft Fur Internationale Zusammenarbeit (GIZ), 1-56. 2016.

[6] S. Am, «TEM:TEM», «COURSA» ET «YASSIR» Taxi online: ça roule... Retrieved February 17, 2019, from 03/09/2018 website: http://www.lexpressiondz.com/actualite/299143-taxi-online-caroule.html, 2018

[7] Y. Bouran, الجزائر.. انتشار واسع لنطبيقات حجز سيارات الأجرة. Retrieved February 17, 2019, from 15/01/2018 website: https://alain.com/article/algeria-wide-spread-taxi-applications, 2018.

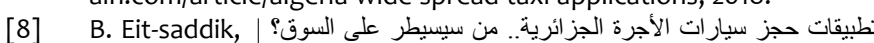
أندرويد ديزاد. Retrieved March 4, 2019, from 10/01/2018 website:

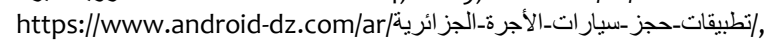
2018.

[9] FromTo, La distance de Alger à Ghardaïa. Retrieved February 17 2019, from 2019 website: https://fromto.city/fr/la-distance-entre-lesvilles/wilaya-d-alger,alger/wilaya-de-ghardaïa,ghardaïa/algérie, 2019

[10] A. Tabti-Talamali, \& Baouni, T, Public transportation in Algiers: Towards a new governance approach. Case Studies on Transport Policy, 6(4), 706-715. https://doi.org/10.1016/j.cstp.2018.08.009, 2018.

[11] A. Muhanna, \& Qusef, A, Using a new set of heuristics in evaluating Arabic interfaces. Journal of King Saud University - Computer and Information Sciences, 2018.

[12] D. Amazouz, Adda-Decker, M., \& Lamel, L, Addressing codeswitching in French/algerian Arabic speech. Proceedings of the Annual Conference of the International Speech Communication Association, INTERSPEECH, 2017-Augus, 62-66, 2017.

[13] A. Allag, \& Amrani, W, «Analyse sociosémiotique des graffitis urbains: cas des deux villes Amizour et Elkseur » Présenté par: Université Abderrahmane Mira - Bejaia, 2018.

[14] N. Saidin, Singh, D., Drus, Z., \& Akramin, M, Culture Centered Design : Reviews on Cultural Factors Influencing Interface design Elements. Pertanika Journal of Scholarly Research Reviews, 3(1), 42-54, 2017.

[15] R.E. Nisbett, The geography of thought: How Asians and Westerners think differently. Free Press, 2004.

[16] M. Benaida, \& Arabia, S, Cross-Cultural Web Design and Education : A Comparison Between Arab Universities and US Universities Based 
on Hofstede Cultural Dimensions. IJCSNS International Journal of Computer Science and Network Security, 18(10), 2018.

[17] R. Alexander, Thompson, N., \& Murray, D, Towards cultural translation of websites: a large-scale study of Australian, Chinese, and Saudi Arabian design preferences. Behaviour and Information Technology, 36(4), 351-363, 2017.

[18] S.T. Shen, Woolley, M, \& Prior, S, Towards Culture-Centred Design. Interacting with Computers. Interacting with Computers, 18(4), 820-852, 2006.

[19] H. Plattner, An Introduction to Design Thinking Process Guide. Institution of Design Stanford. Retrieved from https://dschoolold.stanford.edu/.../designresources/.../ModeGuideBOOTCAMP201 oL..., 2010.

[20] A.K. Hagaman, \& Wutich, A, How Many Interviews Are Enough to Identify Metathemes in Multisited and Cross-cultural Research? Another Perspective on Guest, Bunce, and Johnson's (2006) Landmark Study. Field Methods, 29(1), 23-41.

[21] M.Tohidi, Buxton, W., Baecker, R., \& Sellen, A, Getting the right design and the design right. In Proceedings of the SIGCHI conference on Human Factors in computing systems (pp. 1243-1252), 2006a.
[22] M. Tohidi, Buxton, W., Baecker, R., \& Sellen, A, User sketches: a quick, inexpensive, and effective way to elicit more reflective user feedback. In Proceedings of the 4th Nordic conference on Human-computer interaction: changing roles (pp. 105-114), 2006b.

[23] C. Ambrose, \& Harris, P, Design Thinking (08). In Basics Design Retrieved from https://books.google.com/books?id=9klpFfZDnWgC\&pgis=1, 2010.

[24] J. Ross, \& Gao, J, Overcoming the language barrier in mobile user interface design: A case study on a mobile health app. Retrieved from http://arxiv.org/abs/1605.04693, 2016

[25] A.S. Affairs, for P, System Usability Scale (SUS), Retrieved April 23, 2019, from https://www.usability.gov/how-to-andtools/methods/system-usability-scale.html, 2017.

[26] M.S.A. Aziz, M. S. A., Lindgaard, G., Hamzah, M. S., \& Whitfield, T. A. (2018). The Design and Evaluation of DACADE Visual Tool: Theoretical Implications. Bulletin of Electrical Engineering and Informatics, 7(1), 90-95.

[27] M.S.A. Aziz, M. S. A., Lindgaard, G., Hamzah, M. S., \& Whitfield, T. A. (2017). A Review on the Existing Visual Tools for Design Students. Indian Journal of Science and Technology, 10, 48. 